\title{
Surgical management of acute type A aortic dissection: branch-first arch replacement with total aortic repair
}

\author{
Sean D. Galvin ${ }^{1}$, Nisal K. Perera ${ }^{2}$, George Matalanis ${ }^{2}$ \\ ${ }^{1}$ Department of Cardiothoracic Surgery, Wellington Regional Hospital, Wellington, New Zealand; ${ }^{2}$ Department of Cardiac Surgery, The Austin \\ Hospital, Heidelberg, Australia \\ Correspondence to: A/Professor George Matalanis, Director. Department of Cardiac Suregry, PO BOX 555, Heidelberg, Victoria 3084, Australia. \\ Email: george.matalanis@austin.org.au.
}

\begin{abstract}
Acute type A dissection (ATAAD) remains a morbid condition with reported surgical mortality as high as $25 \%$. We describe our surgical approach to ATAAD and discuss the indications for adjunct techniques such as the frozen elephant trunk or complete aortic repair with endovascular methods. Arch replacement using the "branch-first technique" allows for complete root, ascending aorta, and arch replacement. A long landing zone is created for proximal endografting with a covered stent. Balloon-assisted intimal disruption and bare metal stenting of all residual dissected aorta to the level of the aortic bifurcation is then performed to obliterate the false lumen (FL) and achieve single true lumen (TL) flow. Additional branch vessel stenting is performed as required.
\end{abstract}

Keywords: Aorta; dissection; endovascular techniques

Submitted May 01, 2016. Accepted for publication May 05, 2016.

doi: 10.21037/acs.2016.05.11

View this article at: http://dx.doi.org/10.21037/acs.2016.05.11

\section{Introduction}

Acute type A aortic dissection (ATAAD) is a morbid condition with surgical mortality as high as $25 \%(1,2)$. The traditional approach of open distal anastomosis with or without hemiarch is associated with high rates of persistent false lumen (FL) patency. This exposes the patient to the risk of ongoing end-organ malperfusion and to the formation of complex arch and thoracoabdominal dissection aneurysms. We have described our method of "branch-first arch replacement" previously $(3,4)$. The technique involves establishment of cardiopulmonary bypass (CPB) with serial disconnection and reconstruction of each arch branch (proceeding from innominate to left subclavian; LSCA) using a trifurcation arch graft with a perfusion side-arm port (TAPP graft, Vascutek Ltd., Renfrewshire, Scotland, UK). Following completion of the innominate anastomosis, the perfusion side-arm port is used for selective antegrade cerebral perfusion (ACP). The common stem of the arch graft is proximally translocated to allow for a long landing zone, should a second stage endovascular intervention be required. The following describes our technique with an emphasis on ATAAD.

\section{Interrogation of preoperative imaging}

Understanding the anatomy of the dissection is of paramount importance to the success of the operation (Figure 1). Ideally, a computed tomography (CT) aortogram extending from the angle of the jaw to the thigh is available. Note must be made of the position and the relationships of the true lumen (TL) and FL along the entire aorta. Each branch vessel is interrogated for dissection and the presence of static or dynamic obstruction. Entry and reentry tears and fenestrations are noted. The presence of a pericardial or pleural effusion may represent subacute rupture and the need for establishment of femorofemoral bypass prior to opening the chest. Intraoperative transesophageal echocardiogram (TEE) defines the presence of aortic regurgitation, pericardial effusion, TL and FL flows, and myocardial dysfunction that may represent coronary involvement. Epiaortic US may 


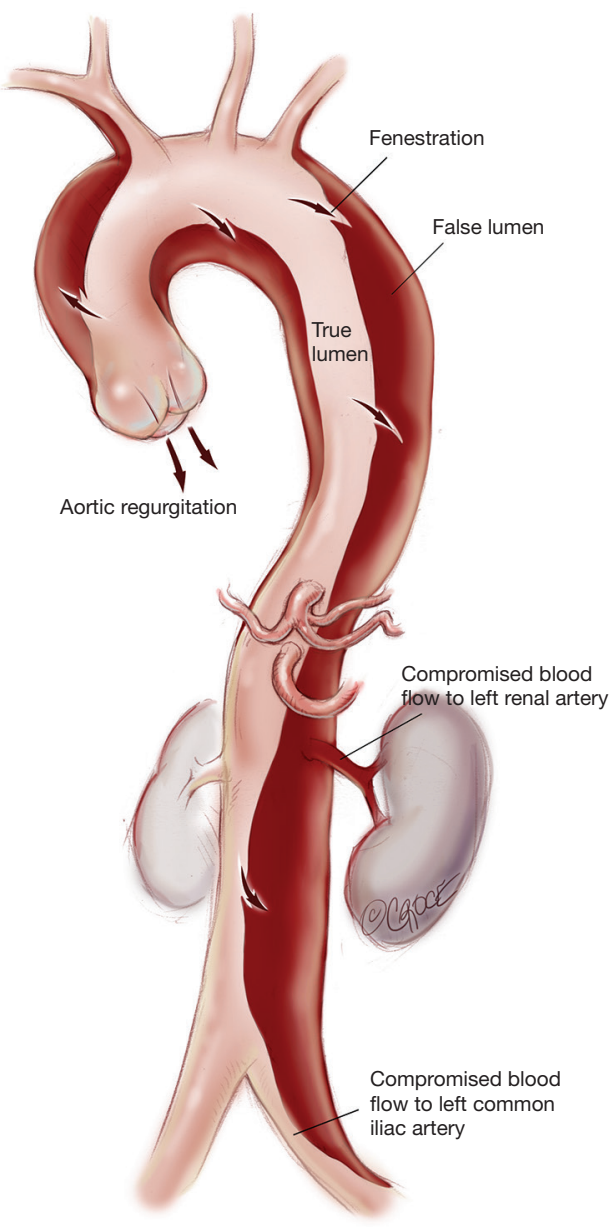

Figure 1 Stanford type A aortic dissection. Note the entry and reentry tears in the ascending, descending thoracic and abdominal aorta. The left renal artery arises from the false lumen, the dissection extend into the left common iliac artery.

be of benefit to the surgeon in assessing TL and FL position in the operative field. Where possible, the case is performed in a hybrid operating suite with access to on-table angiography.

\section{Operative techniques}

\section{Positioning and monitoring}

Bilateral radial or brachial arterial lines are inserted. A femoral arterial pressure line is placed prior to draping, or a femoral sheath can be placed by the surgeon once the operative field is prepared. The monitoring screen is set so that all pressure waveforms are visible and blood pressure can be assessed simultaneously in all major vascular territories.
This is important both for the "branch-first arch" and also to monitor for changes in regional systemic perfusion, an early sign of malperfusion. Cerebral monitoring is carried out by a combination of electroencephalogram bispectral index (BIS), near-infrared spectroscopy (NIRS) and transcranial doppler ultrasonography (TCD). The patient is draped widely to allow access to both groins, the neck, and the anterior chest, should axillary cannulation or carotid intervention be required.

\section{Cannulation and establishment of cardiopulmonary bypass}

The choice of site for arterial cannulation is made following careful assessment of the pre-operative imaging. In the majority of cases, CPB is instituted by femoral arterial inflow and cavo-atrial drainage. Femoral cannulation is via a direct cutdown. Using a seldinger technique the artery is punctured using a Seldinger technique, and the guidewire is confirmed to be in the TL in the descending thoracic aorta on TEE. An elongated one-piece arterial (EOPA) cannula (Medtronic Inc., Minneapolis, MN, USA) is inserted and connected to the arterial circuit. It is important to not advance the femoral cannula too far, as this may cause the tip to be positioned high in the common iliac artery with the loss of collaterals from the pelvis to lower limbs. This is important to reduce the risk of leg ischemia or compartment syndrome. In the setting of a shocked patient, it may be necessary to establish CPB peripherally (femoral artery and femoral vein) prior to opening the chest.

It is important to be alert to the risk of malperfusion during establishment of CPB which may occur with any source of arterial inflow. The transition from pulsatile to non-pulsatile flow with CPB may precipitate preferential FL pressurization and TL collapse. During this time, careful monitoring of the arterial pressure waveforms and interrogation of TL and FL flow on TEE is paramount. Detection includes noting flaccidity of the arch, high line pressures, falling NIRS, reduced TL flow on TEE, and divergence of arterial line monitoring lines which are recorded at multiple sites. Corrective measures can be instituted including changing or adding extra arterial inflow sources. A simple maneuver to reduce the risk of malperfusion during the arch reconstruction is to leave the heart partially full with continued ventilation, so that cardiac ejection contributes to TL flow.

Alternative arterial cannulation sites include: (I) the contralateral femoral artery; (II) the axillary artery; (III) the common carotid artery and (IV) direct TL cannulation of 
the ascending aorta under US guidance using a Seldinger technique. In extreme circumstances when CPB cannot be instituted by other methods; (V) the aorta can be transected under a period of controlled exsanguination with the TL directly cannulated and the aorta clamped proximally; or (VI) direct transapical cannulation can be performed and the cannula positioned across the aortic valve with its tip in the TL of the ascending aorta. In addition, a left ventricular vent is placed via the right superior pulmonary vein and a retrograde coronary sinus cannula is placed for the delivery of retrograde cardioplegia.

\section{Branch-first arch replacement}

We use a modified trifurcation arch graft with a side-arm perfusion port (TAPP graft, Vascutek Ltd., Renfrewshire, Scotland, UK) for the arch reconstruction. This technique allows the innominate artery to be debranched and perfused via the TAPP graft within 10 minutes, ensuring that antegrade TL cerebral perfusion is rapidly secured. A separate head circuit allows control and titration of cerebral flows. Antegrade TL perfusion is provided to each arch branch vessel as the arch reconstruction proceeds and optimal cerebral protection is ensured.

To facilitate arch exposure, the thymus is divided in the midline and the innominate vein is exposed, mobilized and all tributaries ligated. Mobility of the innominate vein allows for exposure of the arch without the need for its ligation or division. Its preservation allows for maximal cerebral venous drainage and avoids left hemispheric venous hypertension which may be a source of cerebral injury. If the clinical situation allows, we prefer to do this initial mobilization and dissection prior to administration of heparin.

Establishment of CPB early allows optimal control of flow during periods of manipulation and clamping of the branch vessels. As the arch itself is intact during the entire period of the arch de-branching, perfusion of both the heart and vital organs is maintained via the systemic bypass circuit. Cardiac ischemic exclusion time is therefore limited and the need for circulatory arrest during arch reconstruction negated.

\section{Innominate reconstruction and establishment of ACP}

Each arch branch is exposed for a length of 3-4 cm using a 'no-touch' technique. The innominate artery is clamped just proximal to its bifurcation and about $1 \mathrm{~cm}$ distal to its origin from the arch and transected between the clamps (Figure $2 A$ ). The distal stump is then anastomosed to the first limb of the TAPP graft in an end-to-end fashion (Figure 2B). The heart is still beating during construction of this anastomosis and the patient is on full flow CPB. Cerebral perfusion is therefore maintained by contralateral flow through the left common carotid artery and the associated collateral channels (4). The graft is de-aired and allowed to back bleed to remove both air and any potential debris from the limbs. The side-arm of the TAPP graft is now connected to a separate cerebral head circuit which is used for antegrade flow (Figure 2B). This allows ACP to be instituted without the need for axillary cannulation. This is a major advantage in the setting of ATAAD as axillary cannulation may be time consuming and technically difficult. In the setting of innominate artery dissection, right axillary cannulation has the potential to create a 'flutter valve' effect across the right common carotid artery and may precipitate right hemispheric cerebral malperfusion. The separate head circuit allows us to alter cerebral flow and perfusion independent of systemic flows. Cerebral flow is adjusted according to changes in cerebral oxygenation or right radial arterial pressure (which now serves as a surrogate measure of innominate and hence right carotid arterial pressure). Cerebral perfusion flow rates of approximately 1 litre per minute $(0.8-1.4 \mathrm{~L} / \mathrm{min})$ are generally used. The proximal innominate stump is now ligated.

\section{Left common carotid and left subclavian anastomosis}

The arch reconstruction then proceeds in a similar fashion with reconstruction of the left common carotid and the LSCA (Figure 2C,D). As each vessel is anastomosed to the TAPP graft, cerebral perfusion flow rates are gradually increased and adjusted to maintain adequate pressure and NIRS. Surgeons often worry about the difficulty of reaching and reconnecting the LSCA. In practice, the progression of debranching from the innominate to LSCA (rather than vice versa) allows progressively more room to reach the subclavian artery. An additional short extension $(1-2 \mathrm{~cm})$ along the anterior border of sternocleidomastoid may also allow for additional exposure of the LSCA. One can also delay the LSCA anastomosis untill distal circulatory arrest, where the decompression allows much more room. Additionally, it may easier to manage issues related to retaining the LSCA on the distal aorta subsequently, either with a carotid-subclavian bypass or simple single branch endovascular methods. If the subclavian artery has not been reconstructed, then it can easily be controlled during the construction of the distal anastomosis with the use of 
A

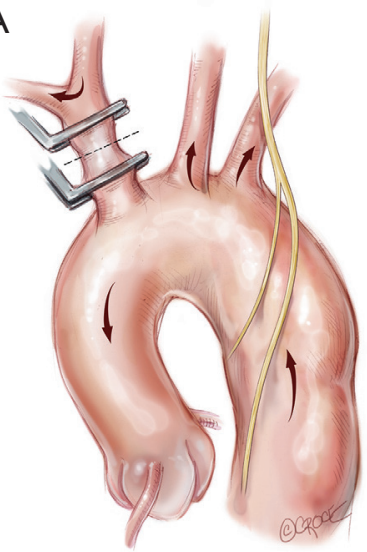

D

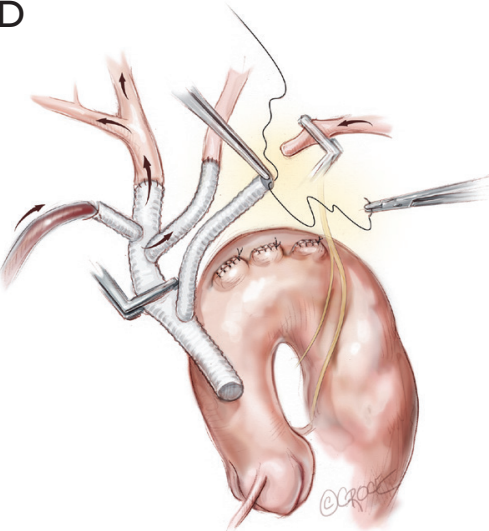

B

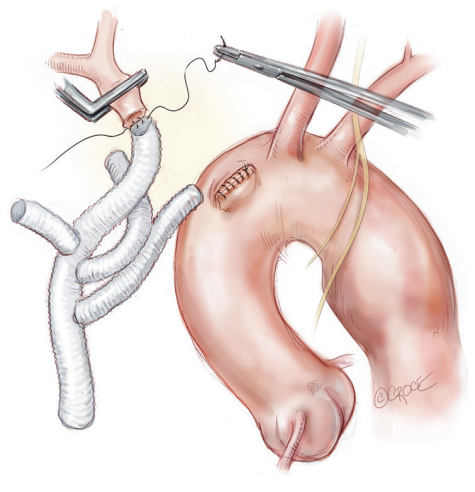

$\mathrm{E}$

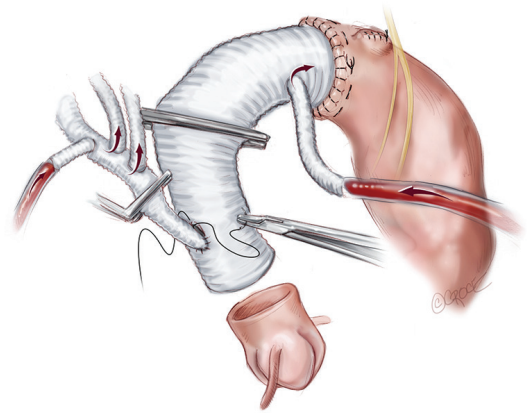

C

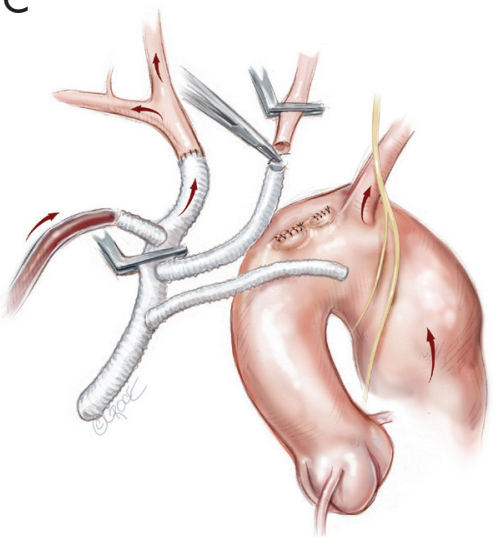

Figure 2 Following establishment of cardiopulmonary bypass, the "branch-first" aortic arch reconstruction proceeds as follows: (A) the innominate artery is clamped proximal to its bifurcation and distal to its origin from the arch. Right hemispheric cerebral perfusion is maintained through the left common carotid and subclavian arteries via collateral channels. Apart from the circle of Willis, there is a plethora of extra-cranial collateral channels that augment cerebral perfusion during individual clamping of branch vessels. These include collateral channels between; the external and internal carotid arteries; the right and the left carotid arteries; the upper and lower body; and the subclavian and carotid arteries (4); (B) the innominate stump is ligated and the anastomosis to the first limb of the branched graft completed; (C) antegrade right hemispheric cerebral perfusion is resumed via the perfusion side arm. Left hemispheric cerebral perfusion during construction of the left common carotid anastomosis is maintained via the same collaterals described above; (D) subclavian anastomosis is completed and all three arch branches are perfused; (E) anastomosis of the arch graft to the distal arch. Note that the distal anastomoses can be performed in Zone 2 which is usually better quality tissues, allows improved access to the anastomoses and reduces the risk of recurrent laryngeal nerve injury. Antegrade flow is recommend via the "ante flow" side arm of the graft. After completion of the root anastomosis, connection of the TAPP graft to the ascending graft proceeds without interruption of cerebral perfusion.

a small Foley balloon catheter placed intraluminally and inflated until back bleeding is controlled.

\section{Distal arch anastomosis}

Even though all three head vessels are debranched, the distal anastomosis can be made in Zone 2 (Figure 2E). This allows easier access to the anastomosis and reduces the risk of left recurrent laryngeal nerve injury. As the cerebral circulation is fully controlled at this stage, an open distal anastomosis can be constructed under moderate systemic circulatory arrest (22-26 degrees Celcius). The distal anastomosis may require reinforcement with external Teflon felt. We avoid the use of tissue glue or an internal (intraluminal) teflon strip. The use of an anteflow graft (Vascutek Ltd., Renfrewshire, Scotland, UK) allows rapid re-establishment of antegrade systemic flow 
on completion of the distal anastomosis. This is important as restarting the systemic circulation from the femoral artery with the arch graft clamped can lead to retrograde pressurization of the FL and may precipitate bleeding at the distal anastomosis.

\section{Proximal reconstruction}

Where possible, we resuspend the aortic valve and perform a simple proximal anastomosis. If the root is extensively dissected or the aortic valve diseased, then a formal root reconstruction is performed, either with a Bentall's, or a reimplantation or remodelling style valve which preserves root replacement.

\section{Positioning of the common stem of the TAPP graft}

The branched graft is passed posterior to the innominate vein, measured and anastomosed to the ascending graft in end-to-side fashion, taking care to avoid redundancy which may lead to kinks or twists, stenosis and an increased risk of thromboembolism (Figure 2E). There is no interruption to cerebral perfusion as the anastomosis is performed proximal to the perfusing side-arm. Placing the TAPP graft on the proximal outer curvature of the ascending graft allows for optimal lie and reduces the possibility of kinking once the chest is closed. Importantly, translocation of the common stem origin of the branch vessels proximally allows for a landing zone of favourable length, should a second stage endovascular reconstruction be required.

\section{Completion of the operation}

Rewarming is completed and hot shot administered. Once the systemic cross clamp is removed, the common stem of the TAPP graft is left clamped, with the cerebral perfusion circuit running until deairing maneuvers are completed. The TAPP graft is then unclamped and the systemic circuit takes over the cerebral circulation. The patient is weaned from bypass, decannulation is performed and hemostasis checked. The soft tissue and pericardium are closed over the prosthetic grafts.

\section{Indications and use of the frozen elephant trunk (FET)}

FET implantation is an important adjunct technique in selected cases. We do not routinely use the FET technique, as the "branch-first arch" replacement described above lends itself to simplified second-stage endovascular intervention if required. Also, the routine use of FET introduces the increased risk of paraplegia, a complication we have not observed with our standard technique. However, we consider FET use in ATAAD with a large entry or re-entry tear at the distal arch and/or proximal descending aorta or a full circumferential dissection at the level of the distal arch.

If a FET is considered, then prior to the arch reconstruction, a guidewire is introduced via a sheath placed in the common femoral artery with its tip positioned above the aortic valve. Wire position is confirmed to be in the TL on TEE. Following the arch debranching, distal circulatory arrest is commenced, the aorta is trimmed back and the anastomosis is usually constructed in Zone 2. This distal anastomosis for a FET typically takes longer (average 40 minutes) than a standard open distal anastomosis (average 25 minutes). Although cerebral perfusion is completely controlled, we typically cool to 20-24 degrees for a FET. The hybrid stent graft, either E-vita (JOTEC GmbH, Hechingen, Germany) or Thoraflex (Vascutek Ltd., Renfrewshire, Scotland, UK) is introduced and deployed into the proximal descending aorta (Figure $3 A$ ). The guidewire facilitates deployment in the TL. Position can also be confirmed by direct angioscopy using a sterile flexible bronchoscope. Both of these hybrid grafts now have a sewing cuff that facilitates construction of the distal anastomosis. The lack of a perfusion side-arm on the EVITA graft necessitates direct cannulation of the Dacron portion or sewing an $8 \mathrm{~mm}$ side-arm graft for reestablishment of antegrade systemic flow. The presence of an "Ante-flo" arm in the Thoraflex graft allows establishment of antegrade systemic perfusion immediately following completion of the distal aortic anastomoses. A cross clamp is applied to the non-stented section proximally. Aortic root reconstruction is performed if necessary and finally proximal translocation of the common stem of the TAPP graft to the non-stented arch graft is completed. Sometimes, this will line up so that the common stem can be connected to the stump of the proximal limb of the thoraflex graft (Figure $3 B$ ). If not, it is connected in a standard fashion and the remaining three side-arms of the Thoraflex graft are ligated and not used.

\section{Completion stent grafting: proximal descending aortic endografting, distal aortic relamination with balloon- induced intimal disruption and bare metal stenting}

More recently we have been performing this procedure in selected patients (5). Typically this is performed following discharge from ICU but before discharge from hospital or within the first 3-6 months after the acute repair. In 

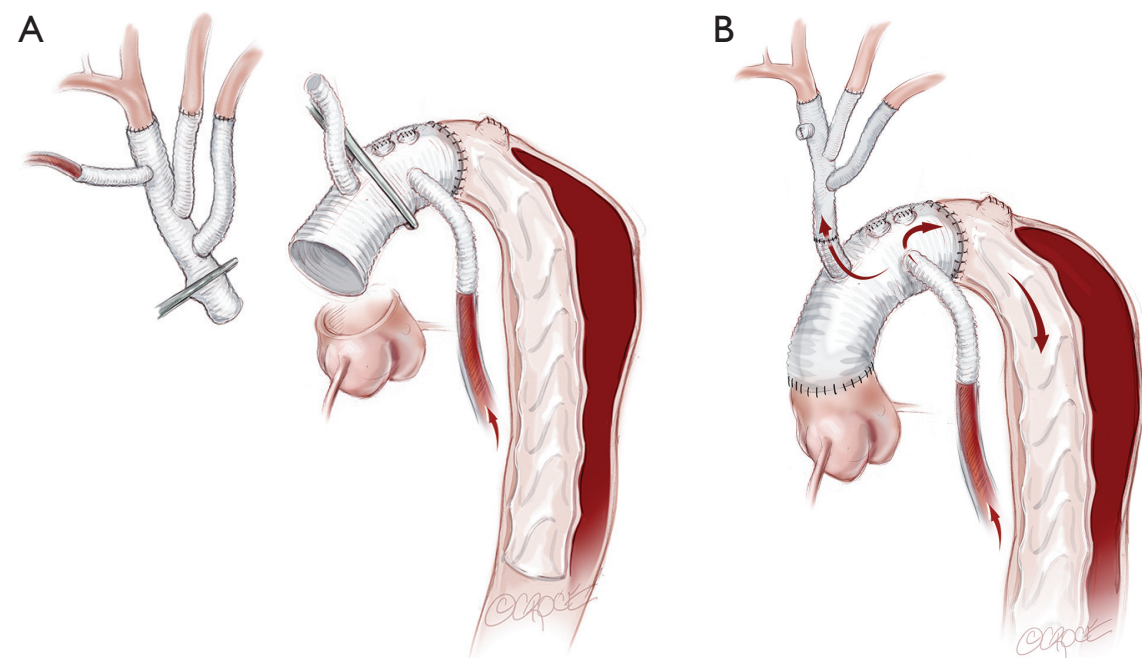

Figure 3 (A) When a FET is used, the "branch first" arch reconstruction proceeds as described above. Under moderate hypothermic arrest, the FET is positioned and deployed in the true lumen in the descending thoracic aorta. The distal anastomosis is again constructed, usually in Zone 2, and antegrade systemic perfusion recommenced; (B) after completion of the root reconstruction, the TAPP graft is then connected proximally. Depending on the lie of the TAPP graft, it may either be connected to the first limb of the FET graft or the anastomosis can be constructed in a similar fashion to Figure 2. FET, frozen elephant trunk.

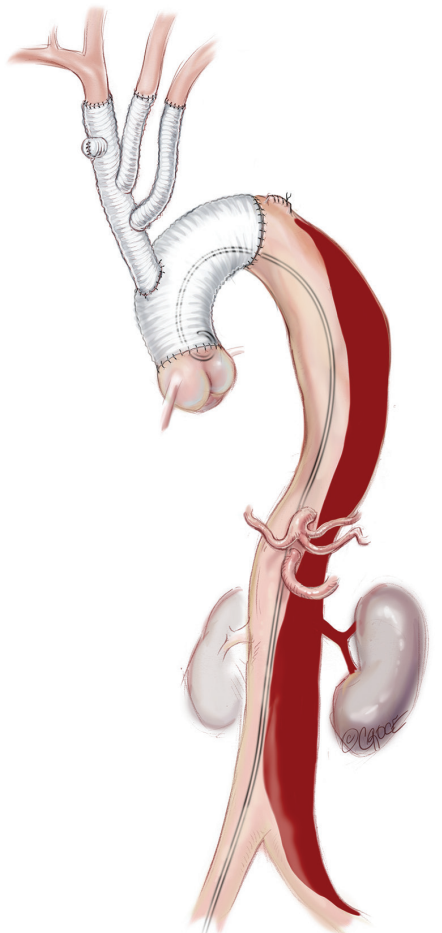

Figure 4 In the case of ongoing or recurrent branch vessel ischemia or malperfusion, radiological TL collapse, rapid dilatation of the FL, or other markers of compromised TL perfusion, we proceed to completion thoracoabdominal stenting. TL, true lumen, FL, false lumen. the setting of early malperfusion, it may be required immediately following the open central repair. We consider completion thoracoabdominal stenting in the following settings:

(I) Ongoing or recurrent branch ischemia with malperfusion;

(II) Radiological TL collapse;

(III) Rapid dilatation of FL;

(IV)Concave TL which is thought to be a marker of compromised TL perfusion and potential future organ ischemia.

\section{Femoral access}

Both femoral arteries are accessed by either open or percutaneous methods (Figure 4). This allows for diagnostic angiography and for stent graft deployment. If a percutaneous method is used, the artery is pre-closed with a percutaneous closure device such as the Proglide (Abbott Vascular Ltd, Redwood City, CA, USA).

\section{Proximal descending aortic endografting with a covered} stent graft

A covered stent graft (Zenith TX2 TAA Endovascular Graft, Cook Medical, Bloomington, IN, USA) is introduced 

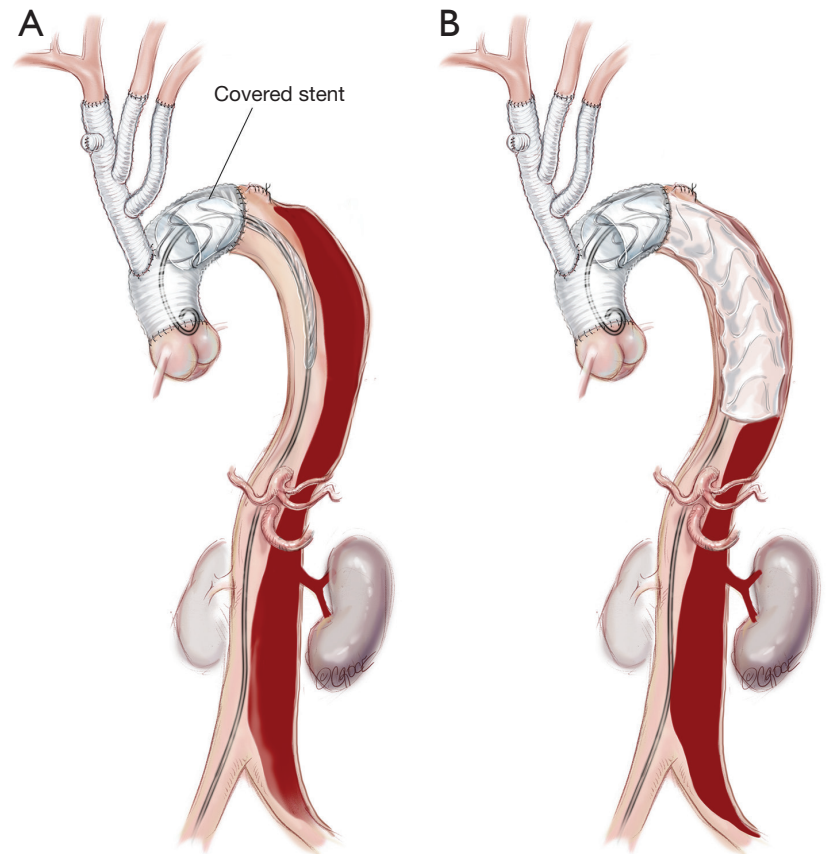

Figure 5 (A) A covered stent graft (Zenith TX2 TAA 112 Endovascular Graft, Cook Medical, Bloomington, IN, USA) is introduced and positioned within the predesigned dacron proximal landing zone; (B) the first stent is deployed so that the distal end is positioned at the junction of middle and upper third of the descending thoracic aorta. Further covered stent grafts can be deployed down to the diaphragm/celiac trunk if required but increased coverage is associated with increased risk of spinal cord injury.

and positioned within the predesigned Dacron proximal landing zone, well in excess of the critical $2 \mathrm{~cm}$ required to achieve a seal (Figure 5). A $10-15 \%$ oversizing is used compared to the surgically inserted Dacron graft. Typically, the first covered stent is deployed so that the distal end lies between the junction of the middle and upper third of the descending thoracic aorta. A further covered stent graft can be used as required and down to the diaphragm or to the level of the celiac trunk, but this is generally avoided to reduce the risk of paraplegia.

\section{Bare metal stenting in residual thoracic and abdominal aorta}

The remaining thoracic and abdominal aorta is then lined with bare metal uncovered stent grafts (Figure 6; Zenith Dissection endovascular stent, Cook Medical Inc.,
A

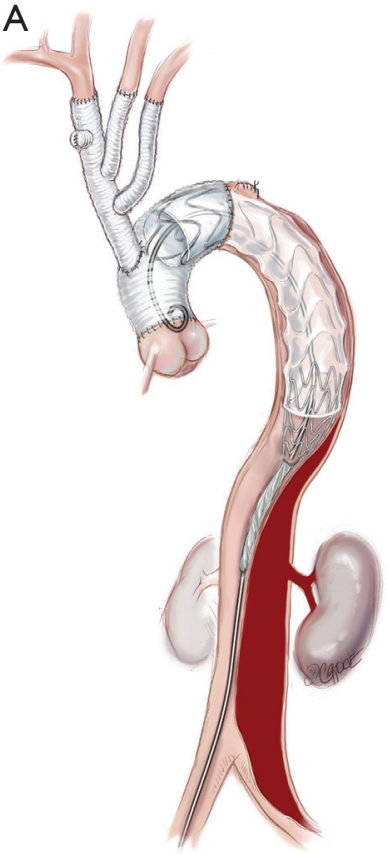

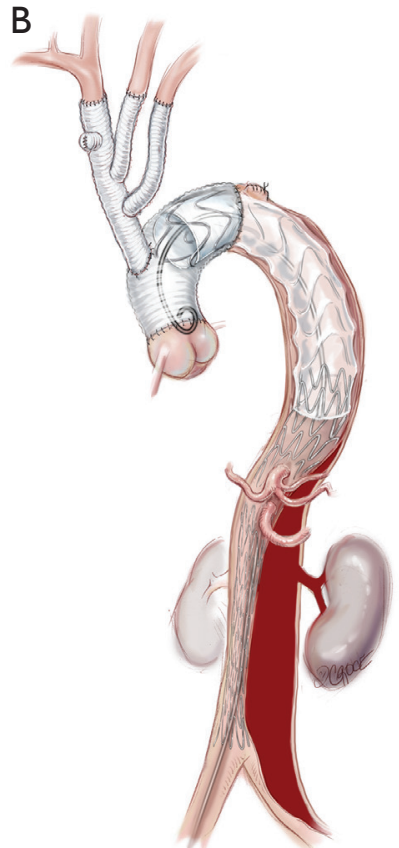

Figure 6 The remaining thoracic (A) and abdominal (B) aorta down to the aortic bifurcation is then lined with bare metal uncovered stent grafts (Zenith Dissection endovascular stent, Cook Medical Inc., Bloomington, IN, USA).

Bloomington, IN, USA). These bare metal stents are positioned with a proximal landing zone $1-2 \mathrm{~cm}$ inside the covered stent graft and deployed sequentially down the TL to the aortic bifurcation. It is important to avoid stent graft overlap in the visceral aorta as this may compromise wire access to the visceral vessels if branch stenting is required.

\section{Distal aortic relamination with balloon-induced intimal disruption}

An angioplasty balloon (Coda Balloon Catheter, Cook Medical Inc., Bloomington, IN, USA) is then used to sequentially expand the bare metal stents and rupture the septum between the TL and FL to create a single aortic channel (Figure 7). The balloon inflation commences at the distal end of the covered stent and moves from proximal to distal along the aorta. It is important not to overinflate the balloon beyond the outer diameter, as this may lead to aortic rupture. The process of restoring a single aortic lumen will usually realign the branch vessels to the TL; however, if this does not occur, one should be prepared to use a stent graft to reestablish flow in a visceral vessel (Figure 8). At the 


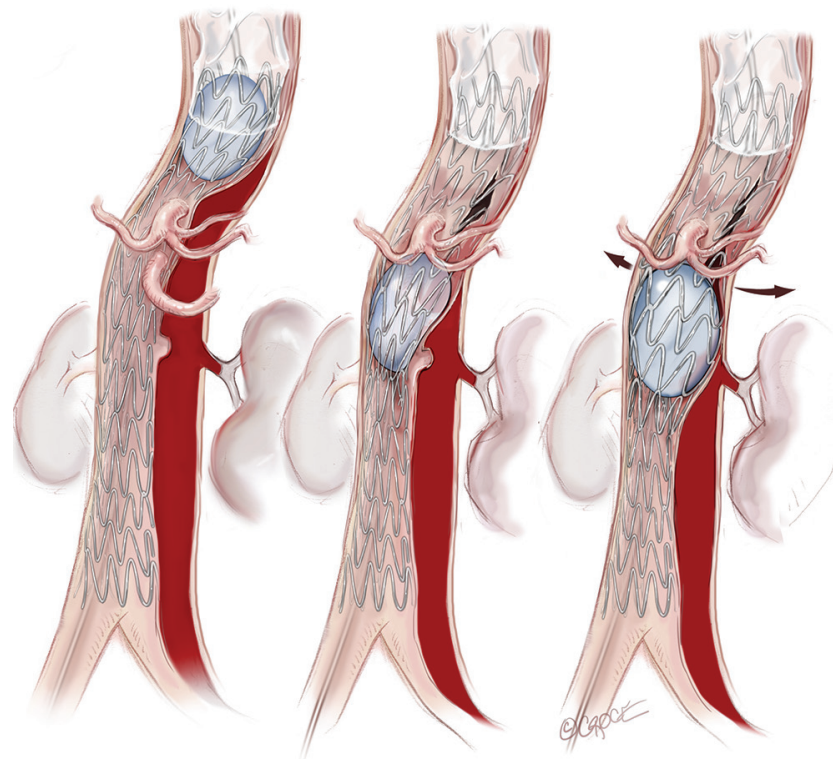

Figure 7 An angioplasty balloon (Coda Balloon Catheter, Cook Medical Inc., Bloomington, IN, USA) is then used to sequentially expand the bare metal stents and rupture the septum between the TL and FL to create a single aortic channel.

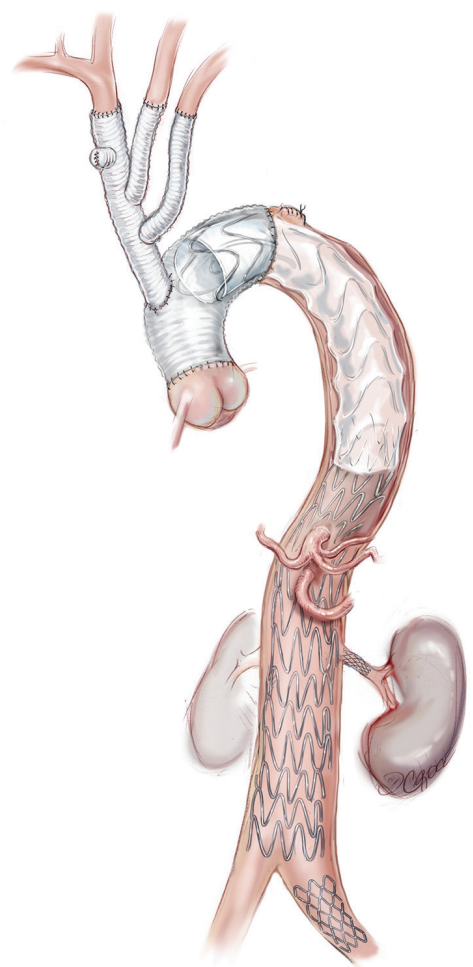

Figure 8 This patient also required stent grafting to the left renal artery and left common iliac artery. At the conclusion of the procedure, aortography is performed and the patency of the aortic lumen and the visceral and other branch vessels are confirmed. conclusion of the procedure, aortography is performed and the patency of the aortic lumen and the visceral and other branch vessels are confirmed.

\section{Comments}

The "branch-first arch" technique has become our standard technique for surgery of the aortic arch and in ATAAD. We have performed this operation with low mortality and with a low incidence of cerebral and other complications (6). Over the last eight years in the setting of ATAAD, we have performed this technique in 51 patients with an average age of 63 years (34-85 years). All three arch branches were replaced in $28(55 \%)$ of the patients and two branches (innominate and left common carotid) in 23 (45\%). The aortic valve was resuspended in 28 (56\%), with the remainder having a root procedure. Elephant trunk was performed in 12 patients, FET in eight (16\%) and standard elephant trunk in four $(8 \%)$. Thirty-day mortality was observed in eight $(15 \%)$ patients. Mortality occurred due to malperfusion and its complications. This led us to a more aggressive approach and the use of total aortic remodeling techniques, as described above. Towards the latter third of the series, mortality was infrequent and eight patients (16\%) proceeded to completion total aortic repair with procedural success achieved in all cases (defined by restoration of uniluminal flow and reversal of malperfusion) and no mortality.

There are a number of potential benefits to performing the "branch-first arch" in patients with ATAAD. ACP is quickly and reliably established, and debranching of the arch with proximal translocation of the common stem of the TAPP graft provides a stable long landing zone for subsequent endovascular stent grafting. It allows for complete root, ascending aorta and arch repair in a safe and reproducible method that removes the need for complex reoperative surgery and simplifies second-stage endovascular intervention. In addition, the technique of total aortic repair described above allows reliable FL obliteration of all dissected thoracoabdominal aorta. In doing so, branch vessel ischemia is treated, TL perfusion is ensured, aortic healing is encouraged while the aorta is provided structural support from the stent graft. A limitation of this technique is the availability of long term follow-up and the uncertainity of stent graft placement in patients with definite or suspected aortopathies. However, we believe that the techniques presented above have the potential to further improve early and late outcomes, effectively manage malperfusion 
and reduce or eliminate the occurrence of late aneurysmal formation.

\section{Acknowledgements}

We would like to thank Beth Croce for her medical illustrations.

\section{Footnote}

Conflicts of Interest: The authors have no conflicts of interest to declare.

\section{References}

1. Hagan PG, Nienaber CA, Isselbacher EM, et al. The International Registry of Acute Aortic Dissection (IRAD): new insights into an old disease. JAMA 2000;283:897-903.

2. Chiappini B, Schepens M, Tan E, et al. Early and late

Cite this article as: Galvin SD, Perera NK, Matalanis G. Surgical management of acute type A aortic dissection: branchfirst arch replacement with total aortic repair. Ann Cardiothorac Surg 2016;5(3):236-244. doi: 10.21037/acs.2016.05.11 outcomes of acute type A aortic dissection: analysis of risk factors in 487 consecutive patients. Eur Heart J 2005;26:180-6.

3. Galvin SD, Matalanis G. Continuous perfusion "Branchfirst" aortic arch replacement: a technical perspective. Ann Cardiothorac Surg 2013;2:229-34.

4. Matalanis G, Galvin SD. "Branch-first" continuous perfusion aortic arch replacement and its role in intraoperative cerebral protection. Ann Cardiothorac Surg 2013;2:194-201.

5. Perera NK, Galvin SD, Brooks M, et al. Total Aortic Repair for Acute Type A Aortic Dissection Complicated by Malperfusion or Symptomatic Branch Vessel Malalignment. Ann Thorac Surg 2016;101:2398-400.

6. Matalanis G, Perera NK, Galvin SD. Aortic arch replacement without circulatory arrest or deep hypothermia: the "branch-first" technique. J Thorac Cardiovasc Surg 2015;149:S76-82. 